\section{Deformaciones cefálicas artificiales en el antiguo Perú}

\author{
Artificial cephalic deformations in the old Perú
}

\section{Resumen}

Las deformaciones cefálicas artificiales en humanos tienen inicio remoto y se difundieron en todos los continentes, en Sudamérica, el Perú constituye el lugar donde se presentaron las formas más variadas e increíbles.

Los motivos por el cual nuestros antepasados realizaban estas deformaciones no es claro y pueden ser muchos, desde los criterios estéticos, éticos, de diferenciación social, económica, moral, mágico, religioso, como divisa de pueblos, etc.

En el Perú se agrupan en dos grandes filums: deformados por llautu y por cuna.

Cada uno de ellos con sus variantes y con sus propias asociaciones culturales, y esta característica les brinda la ubicación histórica correspondiente.

Las deformaciones cefálicas artificiales observadas en los cráneos peruanos produjeron asimetrias craneofáciales alterando no solo las formas sino también su crecimiento y desarrollo, de esta forma producían la acortación de la longitud de estructuras como base de cráneo, maxilares y displasias esqueléticas anteroposteriores.
Artículo de ReVISIÓN

\section{César Andrés Borja Villanueva'y Luis H. Gálvez Calla²}

\author{
Consulta privada, Jr. Pucallipa 286, Breña \\ Lima, Perú \\ 2 Departamento Académico de Ciencias \\ Básicas, Facultad de Odontologia, \\ Universidad Nacional Mayor de San Marcos, \\ Lima, Perú. \\ E-mail: lgalvezc@unmsm.edu.pe
}

Palabras clave: deformación Cefálica Artificial, displasias esqueléticas, asimetría craneofacial.

\begin{abstract}
The artificial cephalic deformations in humans have remote beginning and they spread in all the continents, in South America, the Peru constitutes the place where the most varied and incredible forms were presented.

The reasons for which our ancestors carried out these deformations are not clear and they can be many, from the aesthetic, ethical approaches, of social, economic, moral, magic differentiation, religious, like foreign currency of towns, etc.

In Peru they group in two big filums: deformed by llautu and for cuna. Each one of them with their variants and with their own cultural associations, and this characteristic offers them the corresponding historical location.

The artificial cephalic deformations observed in the Peruvian skulls produced craneofacials asymmetries not altering alone the forms but also their growth and development, this way produced the acortacion of the structures like skull base, maxillary and displasias skeletal anteroposteriores.
\end{abstract}

\section{Introducción}

Ta presencia de las deformaciones cefálicas1 en la humanidad es remota, solo superada por el hecho de pintarse la cara de rojo o de comer sesos del enemigo, ambos procedentes del paleolítico. No existió continente en la tierra que no haya practicado la deformación cefálica, en Asia menor entre la región de los Kurdos y los Juruks, en restos encontrados en Roma, Hungría, Alemania, Inglaterra, etc.

El continente americano no fue ajeno a ello, aquí se enconlró un verdadero mapa de deformaciones1, en el norte los indios Mohave, en el centro el Imperio Maya y sobre todo en el territorio peruano donde se han encontrado los tipos más increíbles.

Cuando se observan estos cráneos causan asombro y es imposible no preguntarse que es lo que intentaban conseguir con ello.

Podríamos empezar por definir al ser humano como un transformador universal, que cambia el medio que lo rodea merced a lo que necesita, que inclusive cambia en si para mejorar sus condiciones de vida frente a lá naturaleza o a sus similares, de esta forma puede realizar cambios en su integridad física ya sea por deformaciones o por complementaciones (prótesis y accesorios), además puede cambiar su personalidad, su tabla de valores, sus conceptos de ética y estética dependiendo de la asociación cullural que lo circunde. Es de notar que el ser humano no a dudado en desfigurarse o afligirse castigos y tormentos en pos de la supremacía estética y moral, de ello
Words key: Artificial Cephalic deformation, skeletal displasias, asymmetry craneofacial. nos podemos fijar en el culto al dolor y las desfiguraciones de las corrientes post-modernas (aretes, tatuajes) y aquellas que buscan la gracia divina mediante tormentos en las corrientes eclesiásticas y patristicas ( santos y beatos).

Por ello la deformación cefálica no significa mucho por sí sola sino en la medida que se asocie culturalmente con otros rasgos, de otro modo sería una moda trivial y no lo que fue, una divisa que se dejaba o acogía en base a ciertas condiciones mágico-religiosas o sociales.

Lo cierto es que para valorar el significado de la plástica cefálica habria no solo que comprender el primitivo gusto humano, sino también vincularlos con su profundo contenido simbólico que le corresponde, este simbolismo 
era mágico entendiéndolo como retigioso de forma tal que también se cataloga como arte, no profano, sino más bien moral, que apuntaba a la máxima idealización de su costumbre llegando al punto de convertirlo en ley. Estas deformaciones que son terroríficas y repugnantes a nuestra sensibilidad, no buscaba "aquella belleza", sino la idealización, la mitificación, por eso la geometrización de las formas naturales, la adecuación de sus figuras a los espacios de su cosmovisión (huacas, picos, volcanes, totems, etc) y la aparición de la plástica en el Perú en tiempos donde aún no existía la zerámica.

\section{Tipología de la deformación cefálica en el Perú}

In el territorio peruano no hubo provincia alguna que no tuviera su propia forma de deformar la cabeza. Al parecer de los investigadores esta tostumbre estaba enraizada y la pracficaban desde que eran recién nacidos ${ }^{2}$. is posible encontrar en una misma olección, en un mismo lugar, en una nisma cultura, variantes y tipos difejentes de deformación, debido a que tos dignatarios tenían moldes propios, ambién existían los moldes populares; for ello la determinación del molde cropio de una región o cultura a tenilo que ser cuantitativo, de esta forma as deformaciones no solo nos sirven jara distinguir épocas y dilucidar reaciones sino también para reconocer $\mathrm{s}^{\text {ategorías sociales } \mathrm{y} \text { algunas veces }}$ eguir sus raices.

fin el Perú hubieron dos grandes flums: las deformaciones por cuna $y$ as deformaciones por llautu, ambos telacionados a las formas como al sis$c^{\text {ema deformador } y \text { a sus asociaciones }}$ $\mathrm{e}^{\mathrm{u} \text { lturales correspondientes; el llautu }}$ if $s$ originario de las culturas serranas y a cuna de los pueblos costeños.

I

a deformación por cuna se remonta c la época pre-cerámica $e$ inclusive cuando los Incas avanzaron hacia la dosta supieron respetar este sistema eformador.

E

I llautu usado en la sierra como $a^{\text {mamento y para el frio, consistía en }}$ marras en la cabeza y sus origines no on claros.

\section{L as deformaciones cefálicas crificiales y la asimetría raneofacial}

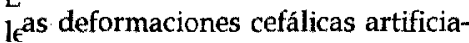
s no son formas geométricas, sino biológicas alteradas. Los cambios morfológicos no son consecuencia exclusiva de las presiones físicas, suponen además las consecuencias biológicas de la comprensión, es lo que se debe de poner como premisa antes de continuar en la explicación. Las deformaciones cefálicas condicionaron la persistencia anormal de algunas suturas (metópica) el cierre prematuro de otros (sagital, borrada desde la infancia en algunos casos) y acciones tróficas sobre cordones nerviosos, vasos sanguíneos, cambios en la presión intracraneal, que repercutieron sobre la ligofisis, alterando el crecimiento y desarrollo craneofacial.

En el año 1964 Ame y Lise Bjork3 realizaron estudios centro geométricos en 149 cráneos serranos deformados por llautu que los autores señalaron como circunferenciales, 31 cráneos costeños deformados por cuna se llamaron simétrico occipital, 23 con deformación occipital izquierda y 21 con deformación occipital derecha, estos dos últimos grupos son pertenecientes al subgrupo Paracas.

\section{Cuadro 1: Tipologia de las deformaciones cefálicas artificiales en el Perá.}
A) Deformados por cuna: asaciado a pueblos costeños, con marcada asimetría cefálica.
Fronto occipital costeño (plagiocefália de la muestra)
a) Precerámico: deformación rústica homogeneidad en la muestra
b) Periodo de culturas locales: variedad en las formas asociado a la trepanación suprainiana.
Inca costeño
Periodo de dominación Inca de gran difusion y promiscuidad cultural

B) Deformados por llautu: asociado a las culturas andinas, no presentan asimetrias cefálicas.

$\begin{aligned} & \text { Subgrupo andino } \\ & \text { (el nombre expresa su } \\ & \text { condición cultural } \\ & \text { deformados solo con llautu) }\end{aligned}$
c) Tipo Pampa: formas andinas extremas

Subgrupos Paracas-Nazca

(a) Tipo Cavemas: la deformación alcanza los huesos (el nombre expresa el ámbito cultural. Deformados por propios de la nariz

llautu más accesorios como

b) Tipo cabeza larga: relacionado con las trepanaciones Paracas

almohadillas y roscas de algodon)
Para determinan la asimetría se uti-

El cráneo humano frecuentemente asimetrias de estructura, los Se llegaron a encontrar resultados sig nicativos, como que la base craneal contras las plastice eran longitudinalmente 列 cráneos no deformados ${ }^{3}$

encontró también que este acortaproporcional a la extensión cráneos con deformación occiHe relacionan de forma directa entre ellos, y la base 
craneal derecha es acortada, también lo será el maxilar y la mandíbula derecha ${ }^{3}$.

La línca media no muestra asimetría en la misma extensión que la base craneal porque el crecimiento de la mandíbula y el maxilar fue menor en el lado deformado. Esta deformación es referido como una asimetría compensatoria de las arcadas a diferencia de la asimetría displásica que ocurre en la Hiperplasia o Hipoplasia unilateral del cóndilo mandibular.

\section{Displasia Esquelética maxilar en cráneos de la Cultura Paracas}

Los hombres Paracas son evidentemente una colectividad costeña, pero usaban sistemas deformadores andinos como lo es el llautu con accesorios de roscas y almohadillas de algodón los que le producian aplanamientos regionales en el cráneo.

La información sobre esta muestra es poca, siendo el trabajo de Richard Koenig4 (1985), único en su género en el Perú; que realizó el análisis cefalométrico en 24 cráneos con sus respectivas mandíbulas, teniendo como objetivo determinar la frecuencia de displasias esqueléticas anteroposteriores (fig. 1,2), para ello se tomó como referencia el plano $\mathrm{S}-\mathrm{N}$ y los ángulos $\mathrm{SNA}, \mathrm{SNB}, \mathrm{ANB}$; cncontrando promedio un ángulo $S N A$ de $86,3^{\circ}$ lo que evidencia una posición adelantada del maxilar con respecto a la base craneal anterior. El ángulo SNB en promedio fue de $77,9^{\circ}$ lo cual indica una posición retruida de la mandíbula con respecto a la base craneal anterior. El valor del ángulo ANB fue de $8,3^{\circ}$ en promedio, indicando una desarmonia maxilomandibular marcada4; concluyendo que existe una alta frecuencia de displasias esqueléticas en estos cráneos y que además estaría localizada en el maxilar superior.

No hubo persona en Paracas que no le deformaran la cabeza, pero no se podría aseverar que los resultados obtenidos en el estudio corresponden al patrón esquelético craneofacial de aquella cultura pan - regional. En cl estudio realizado por Hanzel B. ${ }^{5}(1976)$ comparó cráneos deformados y no deformados con las de personas vivas, $y$ los resultados eran diferentes unos de otros y diferentes también a los datos encontrados por Koenig ${ }^{4}$, pero se debería investigar ampliando el número de la muestra y comparándolos con otros plásticos regionales.

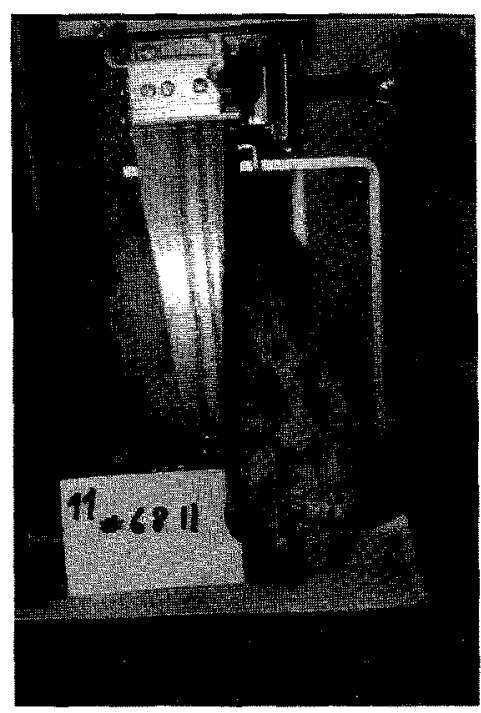

Fig. 1: Cráneo deformado de Paracas con displasia esquelética anteroposterior

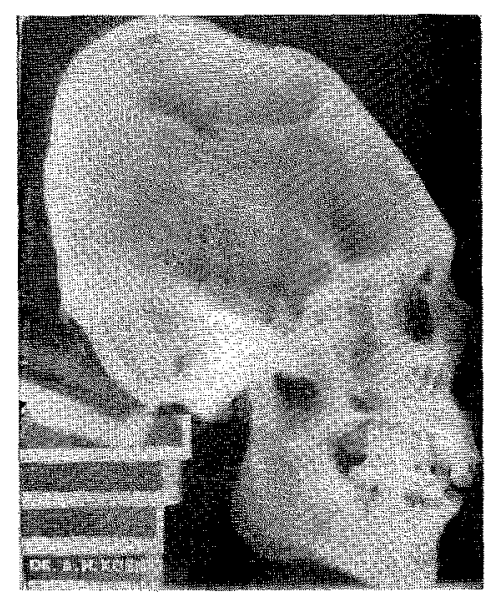

Fig. 2: Cefalografía lateral de cráneo deformado de Paracas.

\section{Conclusiones:}

- Las deformaciones cefálicas artificiales estuvieron presentes en todo el mundo y en el Perú existieron las formas más variadas e increíbles.

- En el Perú las deformaciones cefálicas tuvieron dos grandes filums: el llautu serrano y la cuna costeña.

- Las deformaciones cefálicas artificiales produjeron asimetria craneofacial $y$ alteraron estructuras como base de crâneo, maxilar superior y mandíbula acortándolas en longitud.

- Las alteraciones de las estructuras craneofaciales estuvieron presentes en proporción a la extensión e intensidad de la deformación.

- Las deformaciones cefálicas artificiales correspondientes a la cultura Paracas produjeron displasias esqueléticas anteroposteriores ubicadas en el maxilar superior.

- Las deformaciones cefálicas artificiales alteraron no solo las formas sino la biología, el crecimiento $y$ desarrollo craneofacial.

\section{Referencias}

1. Weiss, Pedro. Osteologia Cultural, $2^{\circ}$ Parte. Tipología de las Deformaciones Cefálicas $1^{\circ}$ ed. Universidad Nacional Mayor de San Marcos. Lima1960

2. Imbelloni, José.Estudios de Morfología Exacta Parte III Rev. del Museo de la Plata T. XXVIII, 1925.

3. Bjork, A, Y Bjork, L. Artificial Craneal deformation and cranio facial asymmetry in ancient peruvians. Journal Dent. Res. 1964, 43: 353-362

4. Koenig Maunsell, Richard. Estudio Cefalometrico Radiouráfico de displasia anteroposterior de los Maxilares en cráneos deformados de la Cultura Paracas. Descriptivo. Tesis Bach UPCH,1985, $53 p$.

5. Hanzei, B. Variation du Profil Craneo - Facial dams les populations Pre colombienne et contemporaines de la cote pacifique sud du Perou. Tesis Doctoral. Universidad Louis Pasteur. Stras Bourgi, 1976, Polonia 